\title{
Epilepsy, headache, and abdominal pain after shunt surgery for idiopathic normal pressure hydrocephalus: the INPH-CRasH study
}

\author{
Jenny Larsson, ${ }^{1}$ Hanna Israelsson, MD, PhD, ${ }^{1}$ Anders Eklund, $\mathrm{PhD},{ }^{2}$ and Jan Malm, MD, $\mathrm{PhD}{ }^{1}$
}

Departments of ${ }^{1}$ Pharmacology and Clinical Neuroscience and ${ }^{2}$ Radiation Sciences, Umeå University, Sweden

OBJECTIVE Adverse events related to shunt surgery are common and might have a negative effect on outcome in patients with idiopathic normal pressure hydrocephalus (INPH). The authors' objectives were to establish the frequencies of epilepsy, headache, and abdominal pain and determine their impact on patient quality of life (QOL), in long-term follow-up after shunt surgery for INPH.

METHODS One hundred seventy-six shunt-treated patients with INPH (mean age 74 years) and 368 age- and sexmatched controls from the population were included. The mean follow-up time after surgery was 21 months (range 6-45 months). Each participant answered a questionnaire regarding present frequency and severity of headache and abdominal pain. Confirmed diagnoses of epilepsy and all prescriptions for antiepileptic drugs (AEDs) before and after shunt surgery for INPH were gathered from national registries. Equivalent presurgical and postsurgical time periods were constructed for the controls based on the date of surgery (the division date for controls is referred to as virtual surgery). All registry data covered a mean period of 6 years (range 3-8 years) before surgery/virtual surgery and 4 years (range 2-6 years) after surgery/virtual surgery. Provoked epileptic seizures were excluded. Patient QOL was assessed with the EuroQoL 5-dimension 5-level instrument.

RESULTS Epilepsy was more common in shunt-treated patients with INPH than in controls ( $4.5 \%$ vs $1.1 \%$, respectively; $p=0.023$ ), as was treatment with AEDs (14.8\% vs $7.3 \%$, respectively; $p=0.010)$. No difference was found between the populations before surgery/virtual surgery (epilepsy, $2.3 \%$ [INPH] vs 1.1\% [control], $p=0.280$; AED treatment, $8.5 \%$ [INPH] vs $5.4 \%$ [control], $p=0.235$ ). New-onset epilepsy and new AED treatment after surgery/virtual surgery were more common in INPH (epilepsy, $2.3 \%$ [INPH] vs $0.0 \%$ [control], $p=0.011 ;$ AED, $8.5 \%$ [INPH] vs $3.3 \%$ [control], $p=0.015$ ). At follow-up, more patients with INPH than controls experienced headache several times per month or more often $(36.1 \%$ vs $11.6 \%$, respectively; $p<0.001$ ). Patients with INPH and unilateral headache had more right-sided headaches than controls $(p=0.038)$. Postural headache was experienced by $16 \%(n=27$ of 169$)$ of the patients with INPH. Twenty percent $(n=35)$ of the patients with INPH had persistent abdominal pain. Headache was not correlated to lower QOL. The study was underpowered to draw conclusions regarding QOL in patients with INPH who had epilepsy and abdominal pain, but the finding of no net difference in mean QOL indicates that no correlation between them existed.

CONCLUSIONS Epilepsy, headache, and abdominal pain are common in long-term follow-up in patients after shunt surgery for INPH and are more common among patients with INPH than in the general population. All adverse events, including mild and moderate ones, should be considered during postoperative follow-ups and in the development of new methods for shunt placement.

https://thejns.org/doi/abs/10.3171/2017.3.JNS162453

KEY WORDS hydrocephalus; normal pressure; ventriculoperitoneal shunt; postoperative complications; headache; epilepsy; abdominal pain

\footnotetext{
ABBREVIATIONS AE = adverse event; $\mathrm{AED}=$ antiepileptic drug; $\mathrm{CRasH}=$ Comorbidity and Risk Factors Associated With Hydrocephalus; EQ5D5L = EuroQoL 5-dimension 5-level instrument; ICD-10-SE = International Statistical Classification of Diseases and Related Health Problems, 10th Revision, Swedish Edition; INPH = idiopathic normal pressure hydrocephalus; MMSE = Mini-Mental State Examination; QOL = quality of life; SNDR = Swedish National Drug Registry; SNPR = Swedish National Patient Registry SUBMITTED September 28, 2016. ACCEPTED March 6, 2017.
}

INCLUDE WHEN CITING Published online September 8, 2017; DOI: 10.3171/2017.3.JNS162453. 
A PPROXIMATELY $80 \%$ of patients with idiopathic normal pressure hydrocephalus (INPH) improve after a CSF shunt insertion. ${ }^{14,37}$ However, adverse events (AEs) related to shunt surgery are common and can negate some of the procedure's positive effects. ${ }^{14}$ Although the frequency of shunt-related AEs has declined, ${ }^{34}$ they still occur in up to $38 \%$ of patients. ${ }^{17}$ In a prospective casecontrol study, serious AEs occurred in approximately $10 \%$ of patients in the first 6 months after their shunt operation. ${ }^{22}$ In previous studies, shunt revision was needed in approximately $13 \%$ of shunt-treated patients with INPH. ${ }^{34}$ Shunt-related AEs are the most common cause of readmission after a neurosurgical procedure ${ }^{33}$ and create high costs and hospital bed utilization. ${ }^{28}$

The main focus of the INPH literature has been on serious $\mathrm{AEs}$ that result in death, hospitalization, or severe disability. ${ }^{9}$ However, mild or moderate AEs, such as pain or epilepsy, can still have a large effect on patient well-being and quality of life (QOL). ${ }^{4,23}$ Headache, epilepsy, and abdominal pain occur after CSF shunt surgery, $6,15,24,26,27,30$ but it is uncertain how often and how much the frequencies in patients with INPH extend above those of elderly patients in the population and whether they are related to the disease or AEs from shunt surgery. Thus, a comparison of these common and subjective symptoms in shunttreated patients with INPH and in age- and sex-matched controls from the general population is needed. However, to our knowledge, no such case-control studies have been performed. Literature regarding epilepsy with INPH is old. ${ }^{24}$ The reports on headache have been focused mainly on headache with low-pressure characteristics, and the frequencies have varied widely. ${ }^{15,26,27}$ To our knowledge, no reports of abdominal pain in patients with INPH exist; abdominal pain has merely been described in other populations. . $^{6,30}$

The objective of this case-control study, part of the INPH-CRasH (Comorbidity and Risk Factors Associated With Hydrocephalus) study, ${ }^{19,20}$ was to determine the frequencies of epilepsy, headache, and abdominal pain in shunt-treated patients with INPH in the long term after surgery and to compare them with age- and sex-matched population-based controls. A secondary objective was to investigate whether these symptoms had an effect on patient QOL.

\section{Methods}

In this case-control study, part of the INPH-CRasH study, ${ }_{19} \mathrm{CSF}-$-shunt-treated patients with INPH were compared with age- and sex-matched controls from the general population. Each participant had a per-protocol visit to his or her caregiver and answered a questionnaire between 6 and 45 months (mean 21 months) after surgery regarding experienced symptoms and severity of symptoms. (Questionnaire available online only; see under Supplemental Information in end matter.) The occurrence of abdominal pain and different types of headache after surgery and information regarding QOL were requested. Information about epilepsy was extracted from national registries that contain data on diagnoses and distributed antiepileptic drugs (AEDs). Registry data were collected for the periods before and after shunt surgery for INPH and for the same time periods for the controls.

\section{Patients}

All patients who undergo shunt surgery in Sweden as a result of hydrocephalus are registered in the Swedish Hydrocephalus Quality Registry. All patients with INPH who underwent shunt surgery between 2008 and 2010 according to registry data were invited to participate in this study. During these years, 5 of 6 Swedish neurosurgical centers contributed to the registry; hence, the registry included approximately $80 \%$ of all shunt-treated patients in Sweden. The study population consisted of $176 \mathrm{CSF}$-shunt-treated patients with INPH, $41 \%(n=73)$ of whom were female. An enrollment flowchart is shown in Fig. 1. The mean age of the patients was 74 years. A ventriculoperitoneal shunt was used in $98.3 \%(\mathrm{n}=173)$ of the patients, and a ventriculoatrial shunt was used in $0.6 \%(\mathrm{n}=1)$. Type of shunt was not specified for $1.1 \%(\mathrm{n}=2)$ of them. Of the shunts used, $84 \%$ had an adjustable valve, and $68 \%$ had an anti-siphon device. On average, the outcome after surgery was favorable for gait ${ }^{32}$ (measured on a scale of 1-8) and modified Rankin Scale score ${ }^{8}$ (activities of daily life evaluation). We found no improvement in Mini-Mental State Examination (MMSE) scores. ${ }^{10}$

\section{Controls}

Age- and sex-matched controls were selected from the Swedish Population Registry, in which all Swedish citizens are registered at birth. The 4 people closest in age and of the same sex as each INPH were invited to participate. The final control population consisted of 368 subjects, $37 \%(\mathrm{n}=135)$ of whom were female (Fig. 1). Their mean age was 73 years.

\section{Exclusion Criteria}

Exclusion criteria were death before 2011 (when the study was initiated), an age of $<60$ or $>85$ years at time of surgery (patients) or when the caregiver visit occurred (controls), and an MMSE score of $<23$ points before surgery (patients) or when the caregiver visit occurred (controls).

\section{Definitions of Events \\ Epilepsy}

The Swedish National Patient Registry (SNPR) contains information regarding diagnoses from all inpatient care in Sweden, presented as International Statistical Classification of Diseases and Related Health Problems, 10th Revision, Swedish Edition (ICD-10-SE) codes for each visit along with discharge dates. It also contains diagnoses from all outpatient care, including acute visits. Diagnoses from primary care are not included. In a previous validation study, the SNPR was shown to have high reliability ${ }^{29}$ The Swedish National Drug Registry (SNDR) contains information regarding all distributed medication prescribed through inpatient and outpatient care, presented as Anatomical Therapeutic Chemical Classification System (ATC) codes along with the dates of prescription and distribution. Any prescribed or distributed AED 


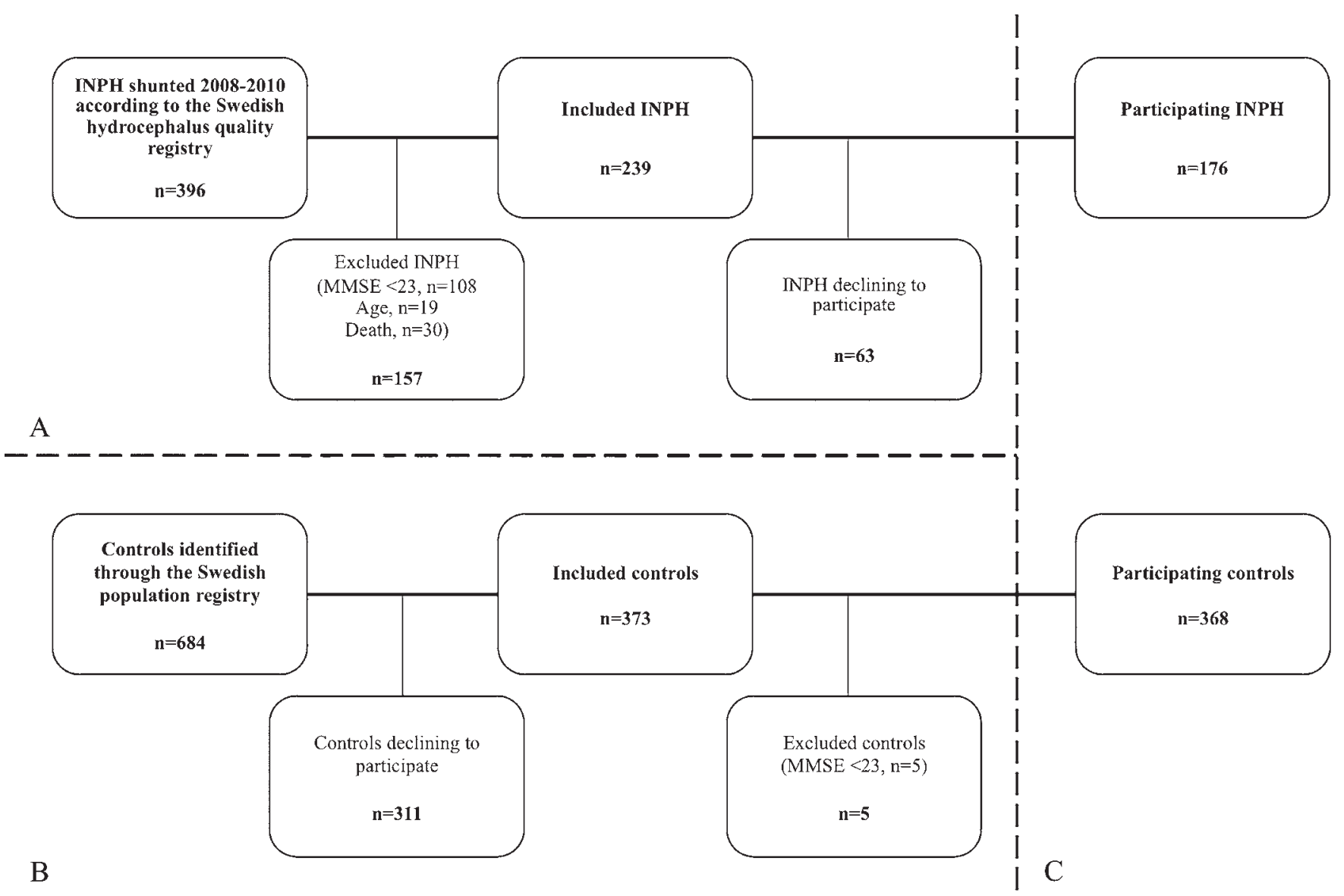

FIG. 1. Flow chart of participating patients with INPH and controls. A: Patients with INPH. B: Controls. C: All participating patients with INPH and controls.

(ATC code N03) according to the SNDR was defined as AED treatment.

Data on diagnoses and treatment with AEDs were collected before and after the date of shunt surgery for all patients with INPH. To enable comparisons between patients and controls, equivalent presurgical and postsurgical time periods were constructed for all controls. The division date for the 2 periods was set to September 5, 2009, for the controls, established through calculation of the date closest in time to the date of shunt surgery of most of the patients with INPH. This division date is referred to here as "virtual surgery." The mean times covered by the registries after surgery/virtual surgery were 3 years (range 2-5 years) for the SNPR and 5 years (range 3-6 years) for the SNDR. The mean times covered by the registries before surgery were 7 years (range 5-8 years) for the SNPR and 5 years (range 3-6 years) for the SNDR. Timelines for gathered data are shown in Fig. 2.

Epilepsy was defined as the presence of an epilepsy diagnosis (ICD-10-SE codes G40.0-G40.9 and G41.0-G41.9) in the SNPR and ongoing treatment with AEDs in the SNDR for at least 6 months. Provoked seizures were excluded. ${ }^{31}$ New-onset epilepsy and new AED treatment during the time period after surgery/virtual surgery were established through the exclusion of participants who had received a diagnosis/treatment before surgery/virtual surgery.

\section{Headache}

In the questionnaire, patients answered questions regarding headache, migraine, unilateral headache, and postural headache (described as headache while in an upright position that disappears while in a horizontal position). The variable "headache" was defined as headache that appeared several times per month or more often. The questions regarding headache were answered by $96 \%(n=169)$ of the patients with INPH and $98.6 \%(\mathrm{n}=363)$ of the controls. One patient who suffered from headache did not answer the question regarding unilateral headache, and the controls were not asked to answer the question regarding postural headache. All the patients with INPH were asked if they had experienced headache of such intensity that it made them visit a hospital (answered by 95.5\% [ $\mathrm{n}=168]$ ).

\section{Abdominal Pain}

The patients answered questions regarding emerging postoperative abdominal pain. The variable "abdominal pain" was defined as pain in the abdominal region that presented after surgery and did not exist before surgery. The question was answered by $98.3 \%(n=173)$ of the shunt-treated patients with INPH an average of 21 months after shunt surgery. Long-term (i.e., persistent) abdominal pain was defined as pain that did not cease during the first months after surgery. Because the question asked for new 


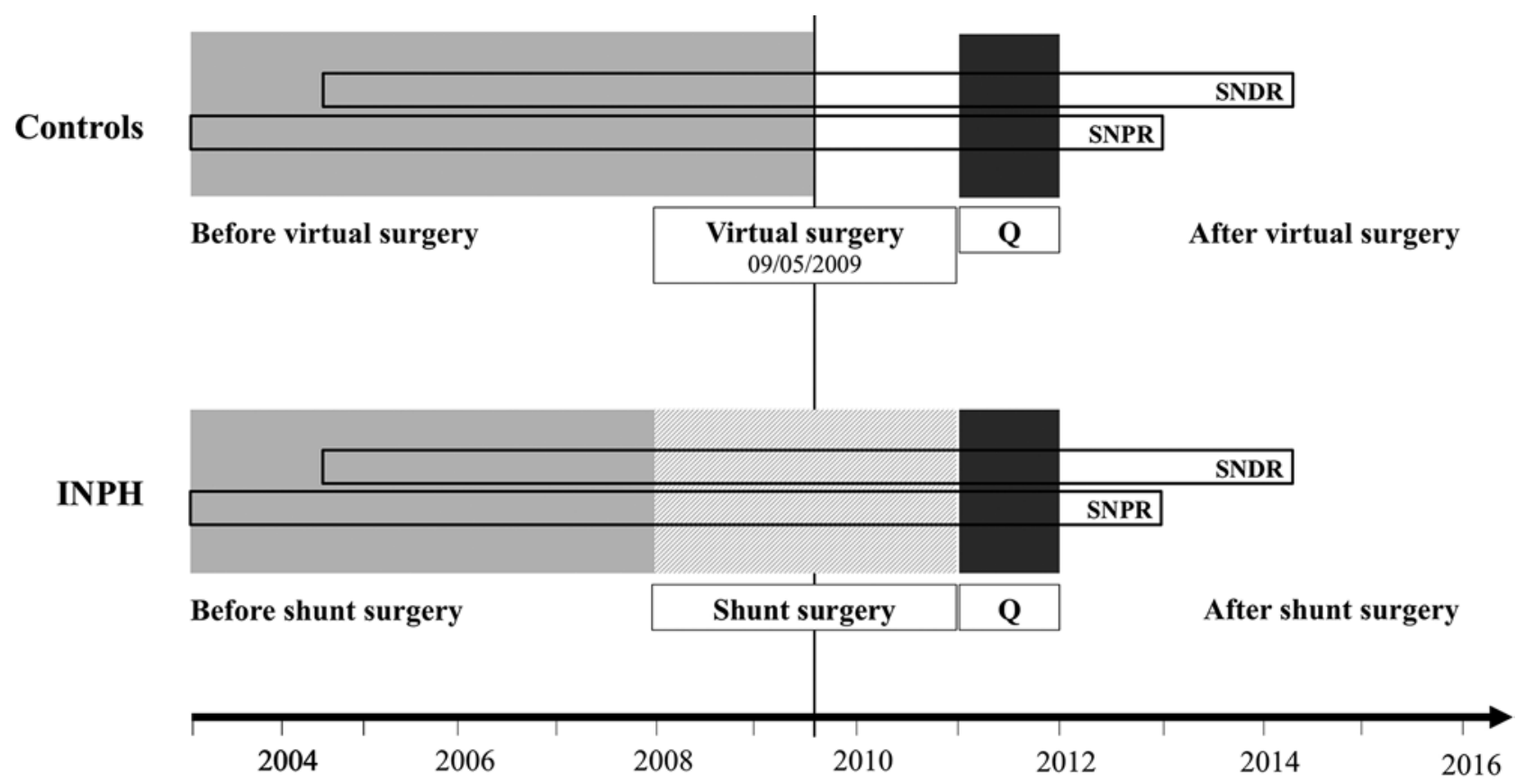

FIG. 2. Data collected from the SNPR and SNDR divided into time frames covering the periods before and after shunt surgery for patients with INPH and the equivalent time periods for controls. Upper: Time frames for controls. Lower: Time frames for patients with INPH. Names of the time periods and research activities are presented underneath each time frame. $Q=$ questionnaire and per-protocol visit to caregiver.

postoperative experiences of pain, the controls were not asked to answer it.

\section{Quality of Life}

To evaluate QOL, the EuroQoL 5-dimension 5-level instrument $(\mathrm{EQ} 5 \mathrm{D} 5 \mathrm{~L})^{7}$ was used. All patients with INPH and all controls answered the EQ5D5L questions. An EQ5D5L index was calculated (https://euroqol.org/wpcontent/uploads/2016/09/EQ-5D-5L_UserGuide_2015. pdf). ${ }^{35}$ In the analysis of how epilepsy and AED treatment affected QOL, all registry data that covered time after the questionnaire had been answered were excluded.

\section{Statistical Analysis}

Statistical analyses were conducted in SPSS Statistics 23 (IBM Corp.). Frequencies of epilepsy, headache, and abdominal pain were calculated for both study populations. To conduct comparative analyses between patients with INPH and controls, the Pearson chi-square or Fisher exact test was used. QOL was compared with an independent-sample t-test between shunt-treated INPH patients and controls with and those without epilepsy, AED treatment, headache, and abdominal pain. We also made comparisons regarding QOL within the populations between subjects with and those without epilepsy, AED treatment, headache, and abdominal pain.

\section{Ethics}

The INPH-CRasH study was approved by the ethical review board at Umeå University. A cover letter informed each participant about the study, and informed consent was obtained from each participant through sub- mission of the questionnaire. Extraction of data from the national registries was approved by the Swedish National Board of Health and Welfare. The INPH-CRasH study has been registered at ClinicalTrials.gov under identifier NCT01850914.

\section{Results}

\section{Frequencies in Patients With INPH and Controls}

Frequencies of epilepsy, AED treatments, headache, and abdominal pain after surgery/virtual surgery are presented in Fig. 3.

\section{Epilepsy}

In general, epilepsy was more common in shunt-treated patients with INPH than in the age- and sex-matched controls (4.5\% vs $1.1 \%$, respectively; OR 4.3, 95\% CI 1.3-14.6; $\mathrm{p}=0.023$, Fisher exact test). Also, a higher percentage of shunt-treated patients with INPH had an AED treatment than did controls $(14.8 \%$ vs $7.3 \%$, respectively; $p=0.010$, chi-square test). When the time periods before and after surgery/virtual surgery were investigated, no presurgical difference between patients with INPH and controls regarding frequency of epilepsy were found $(2.3 \%$ vs $1.1 \%$, respectively; OR 2.1, 95\% CI 0.5-8.6; $\mathrm{p}=0.280$, Fisher exact test). However, after surgery/virtual surgery, more patients with INPH than controls were diagnosed with new-onset epilepsy $(2.3 \%[n=4]$ vs $0.0 \%[n=0]$, respectively; $p=0.011$, Fisher exact test). When treatment with AEDs was investigated, the same pattern emerged. We found no difference in the frequencies of AED treatments between the populations before surgery/virtual surgery 


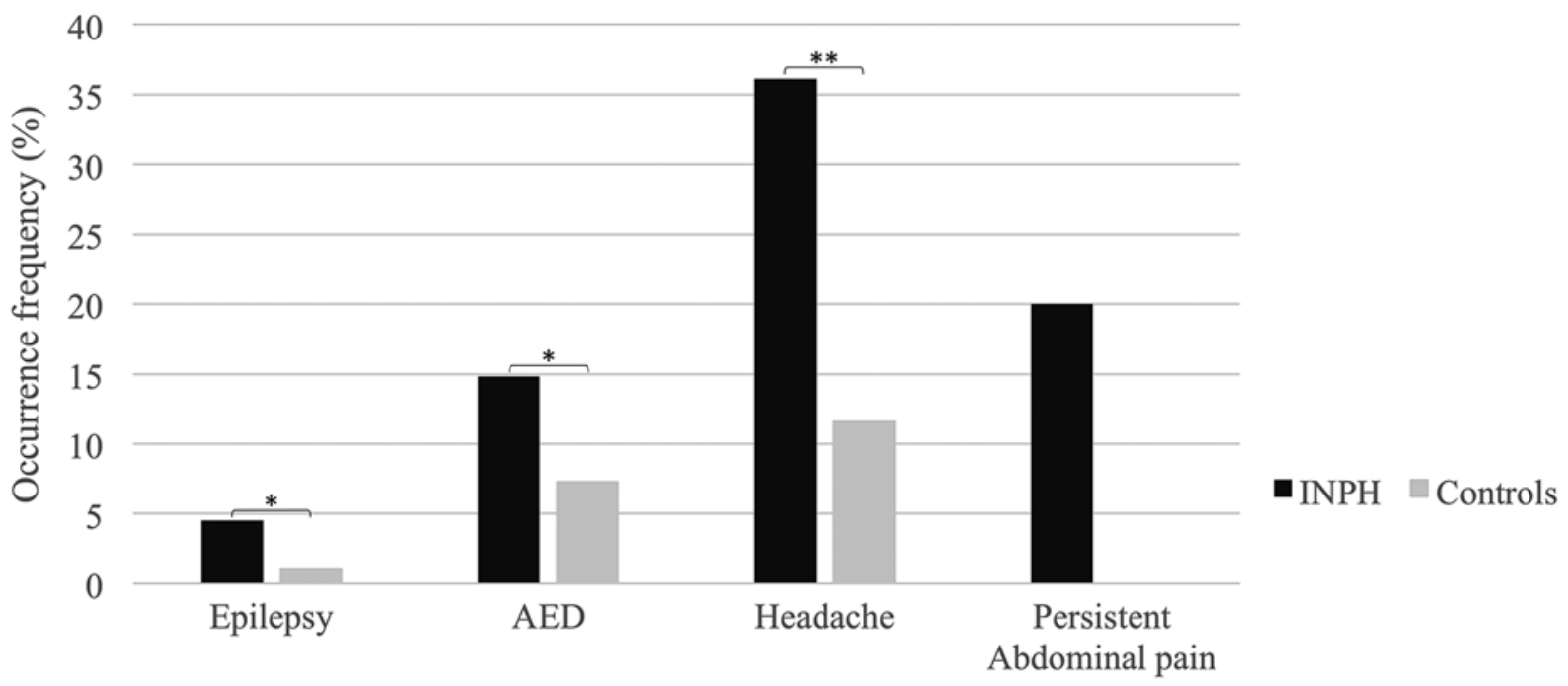

FIG. 3. Epilepsy, headache, and abdominal pain in patients with INPH compared with those in the control population. The bar graph shows the frequencies of epilepsy and AED treatment 2-6 years after shunt surgery/virtual surgery and of headache and persistent abdominal pain 21 months (mean) after shunt surgery/virtual surgery. ${ }^{*} p<0.05$ in comparison between patients with INPH and controls; ${ }^{* *} p<0.001$ in comparison between patients with INPH and controls.

( $8.5 \%$ vs $5.4 \%$, respectively; $\mathrm{p}=0.235$, chi-square test), but a higher frequency of new-onset AED treatments after surgery/virtual surgery among patients with INPH was noted $(8.5 \%$ vs $3.3 \%$, respectively; $\mathrm{p}=0.015$, chi-square test). Epilepsy diagnoses and AED treatments in subjects with epilepsy are plotted in Fig. 4.

\section{Headache}

For details regarding how often headache was experienced, see Fig. 5. Headache, experienced several times per month or more often, was more common in shunt-treated patients with INPH than in controls $(36.1 \%$ vs $11.6 \%$, respectively; $p<0.001$, chi-square test). Regarding postural headache, $44.3 \%(n=27)$ of patients with INPH with headache also experienced postural headache $(16 \%$ of the whole population of patients with INPH). Of all the patients with INPH and postural headache, information regarding type of shunt was available in 26; of these patients, $69.2 \%(\mathrm{n}=18)$ had a shunt with an anti-siphon device, whereas $30.8 \%(n=8)$ did not. We found no correlation between postural headache and absence of an anti-siphon device and no difference in the frequency of programmable shunts in the patients with postural versus nonpostural headache or those with no headache. Also, we found no difference in outcomes after surgery, as measured by gait, modified Rankin Scale score, and MMSE score, between these populations. Of the participants with headache, $51.7 \%(n=31)$ of patients with INPH and $39.5 \%(n=17)$ of the controls experienced unilateral headache (17.6\% of all patients with INPH and $4.6 \%$ of all controls). Patients with INPH with unilateral headache had right-sided headache more often than the controls ( $80.6 \%$ vs $47.1 \%$, respectively; $p=0.038$, chi-square test). Of the shunt-treated patients with INPH, $8.5 \%(n=15)$ had experienced headache of such intensity that they had to visit a hospital. Of those patients, $27 \%(\mathrm{n}=4)$ reported that they underwent radiography that revealed intracranial bleeding.

\section{Abdominal Pain}

Abdominal pain any time after surgery was experienced by $40 \%(\mathrm{n}=70)$ of the shunt-treated patients with INPH. Of those patients, $50 \%(\mathrm{n}=35 ; 20 \%$ of all patients with INPH) had persistent abdominal pain at an average of 21 months after surgery. For details regarding abdominal pain, see Fig. 6.

\section{Quality of Life}

Details regarding QOL in subjects with epilepsy, those who received AED treatment, those with headache, and those with abdominal pain are presented in Table 1 . We found no difference in QOL between the patients with INPH with epilepsy and controls with epilepsy. We also found no difference in QOL between patients with INPH and controls who received AED treatment. When patients with INPH who received AED treatment were compared with those who did not receive AED treatment, no difference was found, but controls who underwent AED treatment had a lower QOL than controls who underwent no AED treatment. For those with epilepsy and those who received AED treatment, the study was underpowered to draw a conclusion regarding the lack of effect on QOL in patients with INPH; however, our finding of no net difference in the mean QOL value for epilepsy indicates the relevance of these results. Regarding headache, patients with INPH with headache had a lower mean QOL than the controls with headache (0.63 vs 0.74 , respectively; $p=0.009$, independent-samples t-test), who in turn had a lower QOL than controls without headache. We found no difference in QOL after surgery between patients with INPH with postural headache and patients with INPH with nonpostural headache or no headache. We also found no net difference in QOL values between patients with INPH with persistent abdominal pain and those without it, although the study did not have the power to reject the possibility of a difference. 


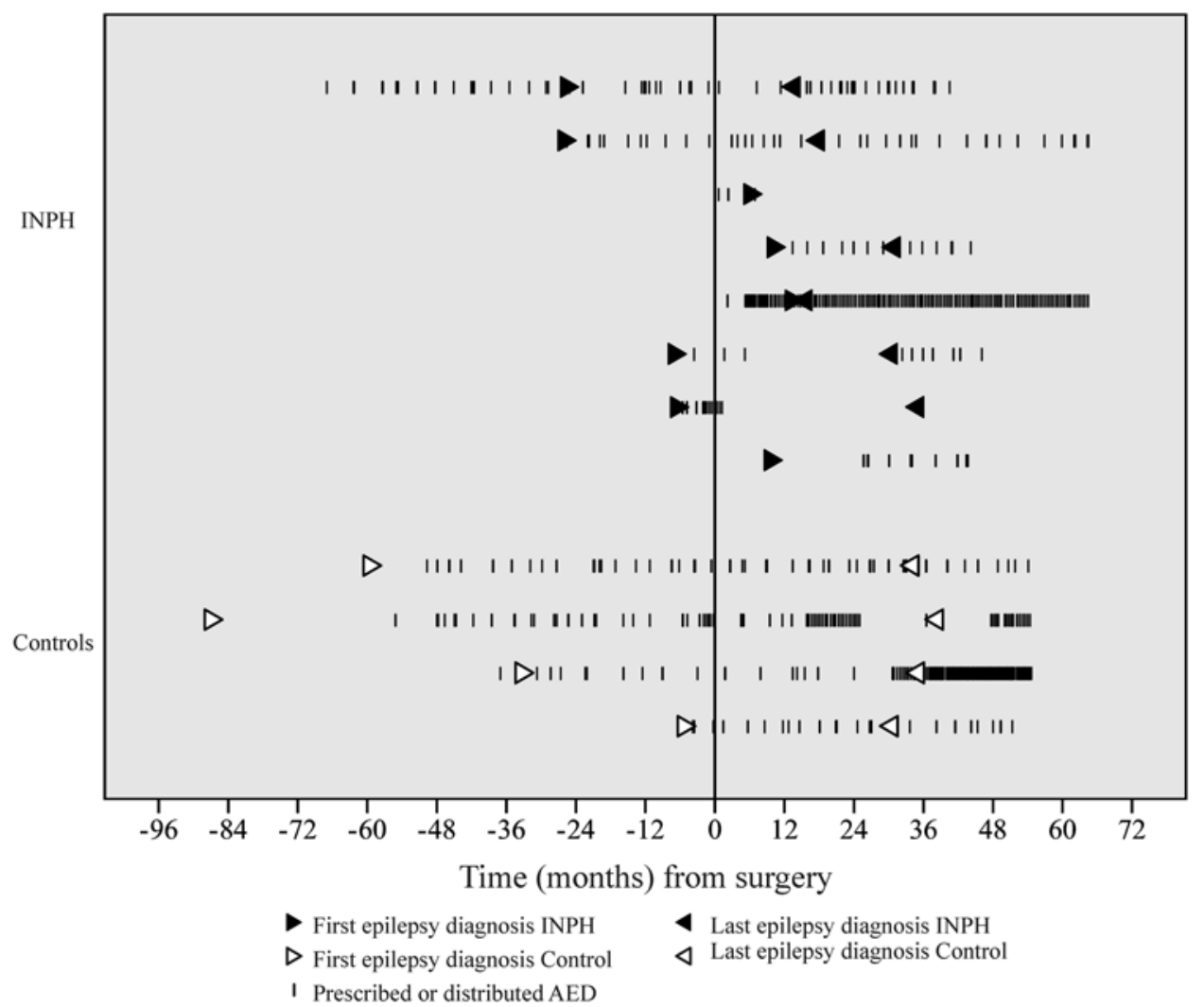

FIG. 4. Timetable of epilepsy diagnoses and AEDs in patients with INPH and controls. The first and last epilepsy diagnoses registered during the time covered by data from the SNPR are indicated with arrowheads, and ongoing AED treatments according to the SNDR in patients with INPH and controls with epilepsy are indicated with vertical lines.

\section{Discussion}

This study is distinguished by its focus on minor and moderate AEs related to CSF diversion for INPH, which is in contrast to many previous studies that focused on serious AEs. ${ }^{17,22,34}$ Also, to our knowledge, this is the first study to have investigated how mild and moderate AEs affect QOL in patients with INPH. The strengths of the study include the population-based setting and the longitudinal design. Our findings show that a diagnosis of epilepsy was 4 times more common in shunt-treated patients with INPH than in age- and sex-matched controls and that AED treatment in the patients with INPH was twice as common; these differences existed exclusively after shunt surgery. In addition, 21 months after surgery, headache
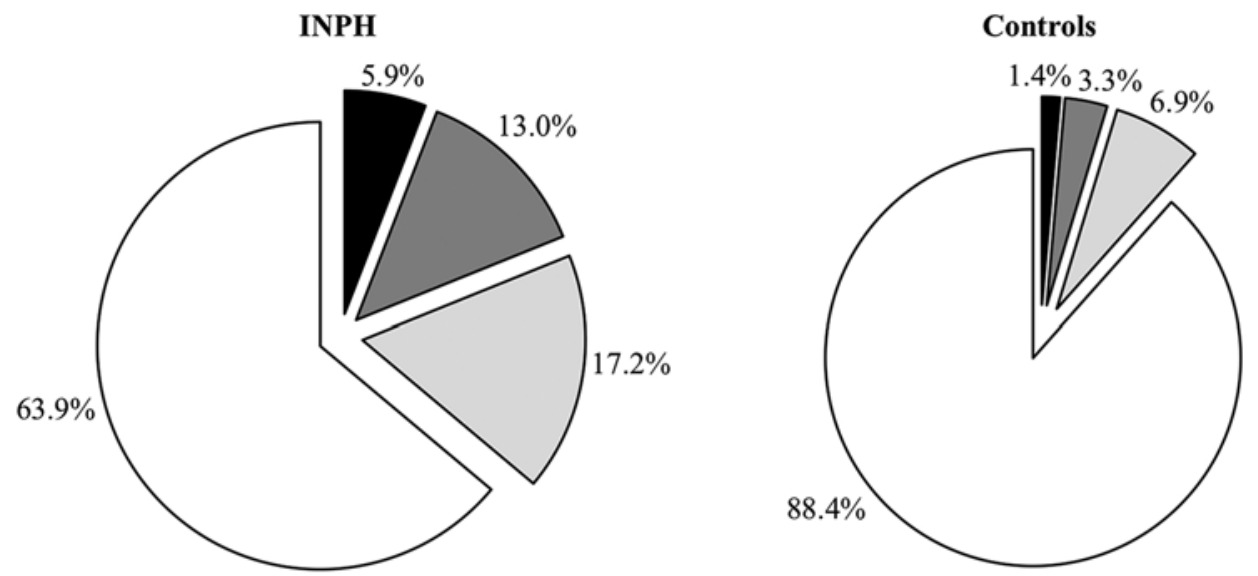

- Every day $\quad \square$ Several times/week

$\square$ Several times/month

$\square$ No headache

FIG. 5. Headache in patients with INPH and controls. The pie chart shows frequencies of headache experienced in patients with INPH and controls 21 months (mean) after shunt surgery/virtual surgery. For those who experienced headache $(36.1 \%$ [INPH] and $11.6 \%$ [controls]), frequencies are highlighted through elevation. 


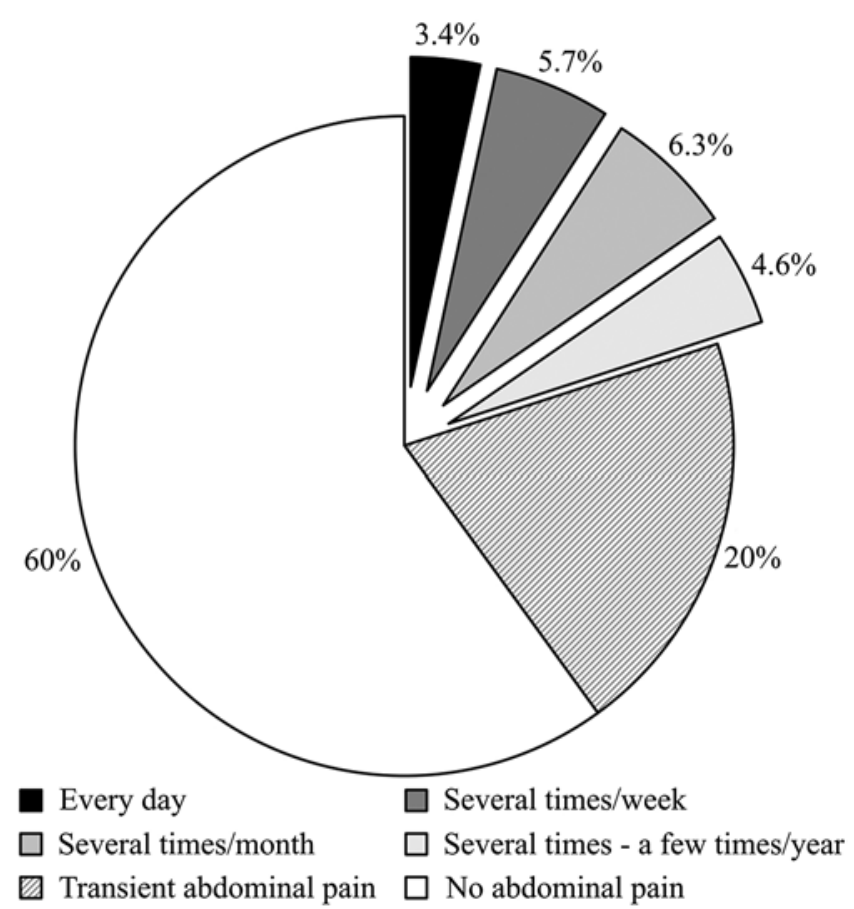

FIG. 6. Persistent abdominal pain in patients with INPH. The pie chart shows frequencies of abdominal pain experienced in patients with INPH 21 months (mean) after shunt surgery, along with frequencies of transient abdominal pain (experienced in the first few months after shunt surgery) and no abdominal pain. Frequencies of persistent abdominal pain are highlighted through elevation.

occurred 3 times as often in shunt-treated patients with INPH than in controls, and one-fifth of the patients with INPH experienced persistent abdominal pain. Headache was not associated with lower QOL in patients with INPH. Only controls with headache and controls who underwent AED treatment had lower QOL scores.

In this study, almost 1 in 20 shunt-treated patients with INPH had a diagnosis of epilepsy, compared with 1 in 100 among the controls. The frequency of epilepsy among the controls was approximately the same as the prevalence found in previous population-based studies $(0.5 \%-$ $\left.1.1 \%^{11,16}\right)$. In previous literature on INPH, postoperative seizures were reported to have various frequencies, $, 1,2,13,18$ but epilepsy was rarely described. In a prospective study more than 25 years ago, the frequency of epilepsy that appeared within 1 year after surgery was $9 \% .{ }^{24}$ Epilepsy can be defined as the occurrence of at least 2 unprovoked seizures or 1 unprovoked seizure with high risk for recurrent seizures. ${ }^{31}$ In our study, we investigated the occurrence of verified epilepsy exclusively. Hence, single seizures or convulsions, common in a perioperative setting, were not included. A recent review paper reported a pooled prevalence of new-onset seizures of $0.7 \% .^{34}$ Thus, the incidence of epilepsy that developed after surgery in our study $(2.3 \%$ of all patients with INPH) must be considered high. Unprovoked seizures in patients with a neurosurgical disease (e.g., traumatic brain injury) are common in elderly patients (relative risk 2.5-4.6, depending on the severity of the injury). ${ }^{25}$ Therefore, shunt insertion is the most likely explanation for the enhanced frequency of epilepsy among
TABLE 1. QOL in patients with INPH and controls after shunt/ virtual surgery*

\begin{tabular}{ccc}
\hline & \multicolumn{2}{c}{ QOL (EQ5D5L Index) } \\
\cline { 2 - 3 } Parameter & Patients w/ INPH & Controls \\
\hline Epilepsy & & \\
\hline Yes & $0.63 \pm 0.2$ & $0.80 \pm 0.2$ \\
\hline No & $0.62 \pm 0.3$ & $0.81 \pm 0.2$ \\
\hline p value & $\mathrm{NS} \dagger$ & $\mathrm{NS} \dagger$ \\
\hline AED treatment & & \\
\hline Yes & $0.52 \pm 0.3$ & $0.66 \pm 0.2$ \\
\hline No & $0.63 \pm 0.3$ & $0.81 \pm 0.2$ \\
\hline$p$ value & $0.074 \dagger$ & 0.002 \\
\hline Headache & & $0.74 \pm 0.2$ \\
\hline Yes & $0.63 \pm 0.2$ & $0.82 \pm 0.2$ \\
\hline No & $0.63 \pm 0.3$ & 0.009 \\
\hline$p$ value & $\mathrm{NS}$ & \\
\hline Abdominal pain & & \\
\hline Yes (persistent) & $0.67 \pm 0.2$ & \\
\hline No & $0.62 \pm 0.3$ & \\
\hline$p$ value & $\mathrm{NS} \dagger$ & \\
\hline
\end{tabular}

NS = not significant

Values are presented as the mean $\pm S D$, except for $p$ values.

* Results are shown for participants with epilepsy, AED treatment, headache, and abdominal pain, and comparisons are made against participants without said characteristics within the same population.

$\dagger$ The study did not have the power to reject the possibility of a difference.

the patients with INPH in our study. This reasoning is supported by our presurgical data, in which no difference was detected between patients with INPH and controls. AED treatment was also more common after surgery and more common among patients with INPH than in the control population, which further strengthens this hypothesis.

We found a large discrepancy between the frequencies of epilepsy and AED treatments (4.5\% and 14.8\% [INPH] vs $1.1 \%$ and $7.3 \%$ [controls], respectively). Initiation of AED treatment after single provoked seizures might be an explanation for this result. In addition, AEDs are sometimes used for pain management or treatment of mental illness. ${ }^{21}$ In our study, pain was clearly overrepresented in the patients with INPH, and we previously reported that depression is a common comorbidity in patients with INPH..$^{19}$

The mean QOL value was not affected by the presence of epilepsy or AED treatment in our study, which was somewhat surprising. However, epilepsy commonly affects a person's daily life in regard to social function, which is influenced by seizure frequency, severity of seizures, depression, AED treatment, ${ }^{4}$ and practical matters such as having a revoked driver's license. Because the number of patients with INPH with epilepsy was low (n $=8$ ), this study was underpowered to draw a conclusion regarding the effect of coexisting epilepsy on QOL in patients with INPH. It is also possible that AED treatment was effective in these cases of epilepsy and, therefore, QOL was not affected.

To avoid subjects with sporadic or occasional headache, 
only those with headache that occurred several times per month or more were investigated. More than one-third of the patients with INPH suffered from headache 21 months after shunt surgery; this high frequency of headache was 3 times more common in patients with INPH than among the controls. We have not found any previous INPH studies that focused on headache in general (presence of undefined headache). The $11.6 \%$ of control subjects who suffered from headache is close to that reported from previous epidemiological studies. Using a definition similar to ours (headache $\geq 2$ times per month), a previous review presented a $17 \%$ prevalence of headache in the general elderly population. ${ }^{3}$ In addition, patients with INPH experienced headache more often. Four times more patients with INPH than controls had headache several times per week or more. Among the controls, the frequency $(4.7 \%)$ of such headache was close to that in a previous epidemiological report on chronic daily headache $(3.9 \%) .{ }^{36}$ In patients with INPH, almost half of those with headache (i.e., $16 \%$ of all patients with INPH) experienced aggravation of headache by postural changes, probably because of their shunt. The frequency of postural headache in patients with INPH that we found corresponds to the higher end of the scale in earlier reports on patients with INPH (range 4\%-15\%). ${ }^{13,15,26,27}$ Previous literature also described findings similar to ours with no difference in overdrainage complication frequencies between patients with a shunt with an anti-siphon device and those with a shunt without an anti-siphon device. ${ }^{12}$ In our study, 1 in 12 patients with INPH reported an episode of newly developed severe headache, and approximately one-fourth of those headaches were caused by intracranial bleeding, according to the patients. Thus, hemorrhage should always be considered a possible diagnosis in shunt-treated patients with INPH who develop acute severe headache.

Headache was also associated with lower QOL in the control population but not in patients with INPH. We have no obvious explanation for this result. It might be that shunt insertion makes patients with INPH more aware of the reason behind their pain, which hypothetically can result in higher acceptance. Or, if more aggressive lowering of valve pressures was needed to resolve the INPH symptoms and caused postural headache, the increased QOL achieved through clinical improvement might have confounded an association between postural headache and lower QOL. This hypothesis is supported by the lack of difference in clinical outcomes between patients with INPH with and those without postural headache. Also, more patients with INPH than controls had right-sided headache. Because the bur hole is commonly placed on the right side of the head, it is plausible that many of the right-sided unilateral headaches were caused by surgical site pain from the shunt insertion. In addition, local scalp tenderness caused by pressure on the periosteum sometimes occurs after shunt surgery and might explain some of these headaches. If scalp tenderness is easier to tolerate than other headaches with measures that release pressure on the scalp, it might have influenced our results on QOL. However, the absence of a difference in QOL values contributes to our theory that headache is a minor AE that is tolerable.

New abdominal pain after shunt surgery, present ap- proximately 21 months after surgery in one-fifth of the INPH population, is not a result of recent surgical incision in the abdomen. It is unlikely that the pain was correlated to the wound, because it should have been completely healed in our patients. Abdominal pain after shunt surgery has not been investigated much before, but reported frequencies range from $0.8 \%$ to $1.5 \% .6,30$ Our frequency is closer to that of patients treated with appendectomy, one-third of whom experience abdominal pain 7 years after surgery. ${ }^{5}$ Also, many of the patients who had abdominal pain (15.4\%) experienced the symptom often (several times per month or more). QOL was not affected statistically, but our study was underpowered to reject differences. Furthermore, different causes for pain probably have different effects on QOL, and more severe pain might be perceived as a bigger problem, possibly big enough to influence QOL. In our study, the severity of abdominal pain probably varied among those afflicted, and cases of less severe pain might have masked a connection with low QOL in cases of more severe pain. We report here a high frequency of subjective abdominal discomfort, and direct irritation from the shunt on intraabdominal structures is a likely explanation.

To characterize the persistence and severity of AEs, additional research is needed. The upcoming Swedish ${ }^{32}$ and American (Adult Hydrocephalus Clinical Research Network [see http://www.ahcrn.org/]) quality registries might be suitable for prospectively researching AEs. Prospective gathering of data would enhance the reliability of future studies and minimize the influence from recollection bias. Such data should include information regarding preoperative and postoperative clinical status, measured with outcome parameters, possible serious and nonserious AEs, and assessments of QOL. Knowledge of predisposing factors for AEs is also needed to optimize the selection criteria for shunt surgery and to establish suitable methods for managing AEs. Hence, information regarding comorbidities and physical status might be useful. We believe that this information should be obtained through visits to a physician, at which standardized interviews and directed physical examinations can be performed.

One limitation in this study is that the information gathered through the questionnaire was subjective, and personal interpretations of the questions were possible. However, pain is a subjective experience, and objective data regarding diagnoses and medications were collected through national registries when possible. Another limitation is that the controls were not asked specifically about postural headache. Therefore, comparative analyses between the patients with INPH and controls could not be performed for this parameter, because when the questionnaire was constructed, it was assumed that controls could not suffer from postural headache. In addition, no information regarding serious AEs was gathered, and thus their influence on QOL could not be evaluated. Regarding the objective registry data, the period before surgery/virtual surgery was longer than the period afterward, which might have resulted in a higher frequency of reported incidences before surgery/virtual surgery. However, this should not influence the comparison between patients with INPH and controls before and after surgery/virtual surgery. One might also argue that errors in the registry data could ex- 
ist as a result of faulty reports from health care facilities, but such errors would exist in both study populations and should not have affected comparisons made between the groups.

\section{Conclusions}

We found a higher frequency of epilepsy and AED treatments in patients with INPH after ventriculoperitoneal shunt surgery than in age- and sex-matched controls. This difference did not exist before surgery. Epilepsy and headache are probably more common after shunt surgery than previous studies have implied, and persistent abdominal pain frequently occurs. Epilepsy, headache, and abdominal pain might be regarded as mild to moderate AEs of which neurosurgeons need to be aware at postoperative follow-up visits and during development of new methods for shunt placement.

\section{Acknowledgments}

We acknowledge Jörgen Andersson for contributing his expertise to the execution of statistical analyses, Kristin Nyman for her assistance in the practical execution of the study, and Sara Qvarlander for her assistance in figure editing.

\section{References}

1. Black PM: Idiopathic normal-pressure hydrocephalus. Results of shunting in 62 patients. J Neurosurg 52:371-377, 1980

2. Boon AJ, Tans JT, Delwel EJ, Egeler-Peerdeman SM, Hanlo PW, Wurzer HA, et al: Dutch Normal-Pressure Hydrocephalus Study: randomized comparison of low- and mediumpressure shunts. J Neurosurg 88:490-495, 1998

3. Bravo TP: Headaches of the elderly. Curr Neurol Neurosci Rep 15:30, 2015

4. Chen HF, Tsai YF, Hsi MS, Chen JC: Factors affecting quality of life in adults with epilepsy in Taiwan: A cross-sectional, correlational study. Epilepsy Behav 58:26-32, 2016

5. Ditzel M, van Ginhoven TM, van der Wal JB, Hop W, Coene PP, Lange JF, et al: What patients and surgeons should know about the consequences of appendectomy for acute appendicitis after long-term follow-up: factors influencing the incidence of chronic abdominal complaints. J Gastrointest Surg 17:1471-1476, 2013

6. Eggenberger ER, Miller NR, Vitale S: Lumboperitoneal shunt for the treatment of pseudotumor cerebri. Neurology 46:1524-1530, 1996

7. EuroQol Group: EuroQol-a new facility for the measurement of health-related quality of life. Health Policy 16:199208, 1990

8. Farrell B, Godwin J, Richards S, Warlow C: The United Kingdom transient ischaemic attack (UK-TIA) aspirin trial: final results. J Neurol Neurosurg Psychiatry 54:1044-1054, 1991

9. FDA: What is a serious adverse event? U.S. Food and Drug Administration. (http://www.fda.gov/Safety/MedWatch/ HowToReport/ucm053087.htm) [Accessed May 5, 2017] 2016

10. Folstein MF, Folstein SE, McHugh PR: "Mini-mental state". A practical method for grading the cognitive state of patients for the clinician. J Psychiatr Res 12:189-198, 1975

11. Forsgren L, Beghi E, Oun A, Sillanpää M: The epidemiology of epilepsy in Europe - a systematic review. Eur J Neurol 12:245-253, 2005

12. Freimann FB, Vajkoczy P, Sprung C: Patients benefit from low-pressure settings enabled by gravitational valves in normal pressure hydrocephalus. Clin Neurol Neurosurg 115:1982-1986, 2013

13. Greenberg JO, Shenkin HA, Adam R: Idiopathic normal pressure hydrocephalus - a report of 73 patients. J Neurol Neurosurg Psychiatry 40:336-341, 1977

14. Halperin JJ, Kurlan R, Schwalb JM, Cusimano MD, Gronseth G, Gloss D: Practice guideline: Idiopathic normal pressure hydrocephalus: Response to shunting and predictors of response: Report of the Guideline Development, Dissemination, and Implementation Subcommittee of the American Academy of Neurology. Neurology 85:2063-2071, 2015

15. Hashimoto M, Ishikawa M, Mori E, Kuwana N: Diagnosis of idiopathic normal pressure hydrocephalus is supported by MRI-based scheme: a prospective cohort study. Cerebrospinal Fluid Res 7:18, 2010

16. Hauser WA, Annegers JF, Kurland LT: Prevalence of epilepsy in Rochester, Minnesota: 1940-1980. Epilepsia 32:429-445, 1991

17. Hebb AO, Cusimano MD: Idiopathic normal pressure hydrocephalus: a systematic review of diagnosis and outcome. Neurosurgery 49:1166-1186, 2001

18. Hughes CP, Siegel BA, Coxe WS, Gado MH, Grubb RL, Coleman RE, et al: Adult idiopathic communicating hydrocephalus with and without shunting. J Neurol Neurosurg Psychiatry 41:961-971, 1978

19. Israelsson H, Allard P, Eklund A, Malm J: Symptoms of depression are common in patients with idiopathic normal pressure hydrocephalus: the INPH-CRasH study. Neurosurgery 78:161-168, 2016

20. Israelsson H, Carlberg B, Wikkelsö C, Laurell K, Kahlon B, Leijon G, et al: Vascular risk factors in INPH: a prospective case-control study (the INPH-CRasH study). Neurology 88:577-585, 2017

21. Italiano D, Capuano A, Alibrandi A, Ferrara R, Cannata A, Trifirò G, et al: Indications of newer and older anti-epileptic drug use: findings from a southern Italian general practice setting from 2005-2011. Br J Clin Pharmacol 79:10101019,2015

22. Kahlon B, Sjunnesson J, Rehncrona S: Long-term outcome in patients with suspected normal pressure hydrocephalus. Neurosurgery 60:327-332, 2007

23. Kim SY, Park SP: The role of headache chronicity among predictors contributing to quality of life in patients with migraine: a hospital-based study. J Headache Pain 15:68, 2014

24. Larsson A, Wikkelsö C, Bilting M, Stephensen H: Clinical parameters in 74 consecutive patients shunt operated for normal pressure hydrocephalus. Acta Neurol Scand 84:475482,1991

25. Mahler B, Carlsson S, Andersson T, Adelöw C, Ahlbom A, Tomson T: Unprovoked seizures after traumatic brain injury: a population-based case-control study. Epilepsia 56:14381444,2015

26. McGirt MJ, Woodworth G, Coon AL, Thomas G, Williams MA, Rigamonti D: Diagnosis, treatment, and analysis of long-term outcomes in idiopathic normal-pressure hydrocephalus. Neurosurgery 57:699-705, 2005

27. Miyake H, Kajimoto Y, Murai H, Nomura S, Ono S, Okamoto Y, et al: Assessment of a quick reference table algorithm for determining initial postoperative pressure settings of programmable pressure valves in patients with idiopathic normal pressure hydrocephalus: SINPHONI subanalysis. Neurosurgery 71:722-728, 2012

28. Patwardhan RV, Nanda A: Implanted ventricular shunts in the United States: the billion-dollar-a-year cost of hydrocephalus treatment. Neurosurgery 56:139-145, 2005

29. Rück C, Larsson KJ, Lind K, Perez-Vigil A, Isomura K, Sariaslan A, et al: Validity and reliability of chronic tic dis- 
order and obsessive-compulsive disorder diagnoses in the Swedish National Patient Register. BMJ Open 5:e007520, 2015

30. Schucht P, Banz V, Trochsler M, Iff S, Krähenbühl AK, Reinert M, et al: Laparoscopically assisted ventriculoperitoneal shunt placement: a prospective randomized controlled trial. J Neurosurg 122:1058-1067, 2015

31. St. Louis EK, Cascino GD: Diagnosis of epilepsy and related episodic disorders. Continuum (Minneap Minn) 22 (1 Epilepsy):15-37, 2016

32. Sundstrom N, Malm J, Laurell K, Lundin F, Kahlon B, Cesarini $\mathrm{KG}$, et al: Incidence and outcome of surgery for adult hydrocephalus patients in Sweden. Br J Neurosurg 31:21-27, 2017

33. Taylor BE, Youngerman BE, Goldstein H, Kabat DH, Appelboom G, Gold WE, et al: Causes and timing of unplanned early readmission after neurosurgery. Neurosurgery 79:356369, 2016

34. Toma AK, Papadopoulos MC, Stapleton S, Kitchen ND, Watkins LD: Systematic review of the outcome of shunt surgery in idiopathic normal-pressure hydrocephalus. Acta Neurochir (Wien) 155:1977-1980, 2013

35. van Hout B, Janssen MF, Feng YS, Kohlmann T, Busschbach J, Golicki D, et al: Interim scoring for the EQ-5D-5L: mapping the EQ-5D-5L to EQ-5D-3L value sets. Value Health 15:708-715, 2012

36. Wang SJ, Fuh JL, Lu SR, Liu CY, Hsu LC, Wang PN, et al: Chronic daily headache in Chinese elderly: prevalence, risk factors, and biannual follow-up. Neurology 54:314-319, 2000

37. Williams MA, Malm J: Diagnosis and treatment of idiopathic normal pressure hydrocephalus. Continuum (Minneap

Minn) 22 (2 Dementia):579-599, 2016

\section{Disclosures}

This study was supported financially through a regional agreement between Umeå University and the Västerbotten County Council (ALF). Financial support was provided by the fund for Cutting Edge Medical Research and by the Research and Development Fund, both granted by the County Council of Västerbotten. Dr. Eklund was granted financial support by the Swedish Research Council. The abovementioned organizations are governmental or institutional organizations with no practical involvement or economic interest in the study.

\section{Author Contributions}

Conception and design: Israelsson, Eklund, Malm. Acquisition of data: Israelsson. Analysis and interpretation of data: all authors. Drafting the article: Larsson. Critically revising the article: all authors. Reviewed submitted version of manuscript: all authors. Statistical analysis: Larsson. Study supervision: Eklund, Malm.

\section{Supplemental Information \\ Online-Only Content}

Supplemental material is available with the online version of the article.

Questionnaire. https://thejns.org/doi/suppl/10.3171/2017.3. JNS162453.

\section{Correspondence}

Jenny Larsson, Department of Pharmacology and Clinical Neuroscience, Norrlands University Hospital, Umeå University, SE-901 85 Umeå, Sweden. email: jenny.ma.larsson@umu.se. 